\title{
Predicting Factors of Angiographic Aneurysm Occlusion after Treatment with the Woven EndoBridge Device: A Single-Center Experience with Midterm Follow-Up
}

\author{
(D). Cagnazzo, (D) R. Ahmed, (D) R. Zannoni, (D) Dargazanli, (D).-H. Lefevre, (D) G. Gascou, (DI. Derraz, (D). Riquelme,
}

(D) A. Bonafe, and (D) V. Costalat

\begin{abstract}
BACKGROUND AND PURPOSE: Flow disruption with the Woven EndoBridge is increasingly used for the treatment of intracranial aneurysms. We examined factors leading to aneurysm occlusion and Woven EndoBridge shape change during a midterm follow-up.
\end{abstract}

MATERIALS AND METHODS: Patients with a minimum 12-month angiographic follow-up were included. Through a univariate and multivariate analysis, independent predictors of adequate occlusion (Raymond-Roy 1/Raymond-Roy 2) and Woven EndoBridge shape change (decrease of the height of the device) were assessed.

RESULTS: Eighty-six patients/aneurysms were included. The aneurysm mean size was $5.5 \mathrm{~mm}$ (range, 3-11.5 mm). The most common locations were the MCA $(43 / 86=50 \%)$, basilar tip $(13 / 86=15.1 \%)$, and anterior communicating artery $(12 / 86=14 \%)$. Twenty-one patients $(21 / 86=24 \%)$ had acute SAH. Immediate and long-term Raymond-Roy 1/Raymond-Roy 2 occlusion rates were 49\% (42/86) and $80 \%$ (68/86), respectively. Woven EndoBridge shape change was detected among 22\% (19/86) of cases. At binary logistic regression, wide ostium $(\geq 4 \mathrm{~mm})(\mathrm{OR}=0.2 ; 95 \% \mathrm{Cl}, 0.01-1 ; P=.04)$ and regular aneurysm morphology $(\mathrm{OR}=5.9 ; 95 \% \mathrm{Cl}, 1.4-24$; $P=.01)$ were independent factors of incomplete and adequate aneurysm occlusion, respectively. In addition, irregular morphology $(\mathrm{OR}=5.4 ; 95 \% \mathrm{Cl}, 1.4-19 ; P=.01)$ and a wide ostium $(\mathrm{OR}=9.8 ; 95 \% \mathrm{Cl}, 1.6-60 ; P=.03)$ significantly increased the probability of the Woven EndoBridge shape change. Decrease of the Woven EndoBridge height was more common among incompletely occluded aneurysms $(6 / 12=50 \%$ versus $13 / 74=17.5 \%)$, but it was not an independent prognosticator of occlusion at the multivariate model.

CONCLUSIONS: The likelihood of good occlusion was 5 times lower in the presence of a wide ostium, whereas aneurysms with regular morphology were 6 times more likely to be occluded. Woven EndoBridge shape modification was strongly influenced by the aneurysm shape and ostium size, and it was not independently associated with the angiographic occlusion.

ABBREVIATIONS: AcomA = anterior communicating artery; BT = basilar tip; RR = Raymond-Roy

$\mathbf{T}$ he efficacy and safety of the Woven EndoBridge device (WEB; Sequent Medical, Aliso Viejo, California) have been largely evaluated by several multicentric trials. ${ }^{1}$ Treatment with the WEB resulted in approximately $80 \%$ of complete/near-complete occlusion in many published series. ${ }^{1-3}$ However, as reported in some recent studies, ${ }^{2,4}$ aneurysm recurrence and WEB compaction may occur in nearly $8 \%$ and $40 \%$ of patients, respectively. Despite the increased operator experience and the better patient selection, factors leading to aneurysm occlusion, recanalization,

Received June 10, 2019; accepted after revision August 5.

From the Neuroradiology Department, University Hospital Güi-de-Chauliac, Centre Hospitalier Universitaire de Montpellier, Montpellier, France.

Please address correspondence to Federico Cagnazzo, MD, Neuroradiology Department, CHU Gui De Chauliac, 80 Ave Augustin Fliche, 34000 Montpellier, France; e-mail: f.cagnazzo86@gmail.com

Indicates article with supplemental on-line appendix and tables.

http://dx.doi.org/10.3174/ajnr.A6221 or WEB shape modification are not fully understood. Very few studies explored patient and aneurysm characteristics potentially associated with the angiographic results after WEB implantation. Our study aimed to investigate independent predictors of adequate aneurysm occlusion and WEB shape modification among 86 patients/aneurysms available during midterm angiographic follow-up.

\section{MATERIALS AND METHODS \\ Patient Selection}

Our hospital institutional review board approved this retrospective study. A prospectively maintained data base of WEBs (20142019) was retrospectively reviewed by 2 , and in case of inconsistency, by 3 investigators independently. We included patients with aneurysms (unruptured or ruptured) treated with the WEB and available at midterm angiographic follow-up (at least 12 months). To assess factors associated with midterm aneurysm 
occlusion, we excluded patients with a radiologic follow-up of $<12$ months. Recanalized aneurysms for which the WEB was used as retreatment strategy were excluded. Indications for treatment were made by multidisciplinary consensus (vascular neurosurgeons, interventional neuroradiologists).

\section{Indications for WEB}

The selection of aneurysms treated with the WEB was performed by interventional neuroradiologists according to aneurysm characteristics and available sizes of the device. The main indications for WEB treatment were the following: 1) unruptured and ruptured wide-ostium aneurysms difficult to treat with simple coiling; 2) aneurysms located at bifurcation points not amenable to treatment with coiling or balloon-assisted coiling and where a flow diverter may lead to the risk of covering bifurcation branches or perforators. In addition, due to the progressive enlargement of WEB indications ${ }^{5}$ and on the basis of our own positive results, some small-neck or small-sized aneurysms were treated with the WEB.

\section{Antiplatelet Therapy}

For unruptured aneurysms, patients were premedicated with dual antiplatelet therapy in case of a strategy shift to stent-assisted technique (aspirin, $75 \mathrm{mg}$, and clopidogrel, $75 \mathrm{mg}$, starting 5 days before treatment). Daily aspirin administration was continued for 1 month. In case of additional stent placement, the dual antiplatelet therapy was maintained for 3 months, and on the basis of the clinical and radiologic evaluation, the patients were switched to aspirin. The VerifyNow P2Y12 assay (Accumetrics, San Diego, California) was used to test the platelet inhibition (P2Y12 reaction unit). Concurrent with the procedure, intravenous heparinization was performed (activated clotting time of $>250$ seconds).

For ruptured aneurysms, apart from heparin in the pressure bags for flushing (1000 IU/L), no antiplatelet therapy was used. In case of stent placement, an intravenous bolus of abciximab $(0.125 \mathrm{mg} / \mathrm{kg})$ was administered before stent deployment, and standard dual antiplatelet therapy was started the day after.

\section{Description of Technique}

With the patient under general anesthesia, via a transfemoral approach, access to the aneurysm was obtained in a triaxial fashion. Through a long femoral sheath, a $6 \mathrm{~F}$ guiding catheter was advanced into the carotid artery. The WEB was delivered under roadmap guidance through the dedicated VIA microcatheter (Sequent Medical). Most of the implanted WEBs were singlelayer (SL): accordingly, we included only SL devices. VasoCT (Philips Healthcare, Best, the Netherlands) with diluted iodinated contrast medium was used to assess WEB apposition.

\section{Selection of WEB Size}

Size selection derived from the measurements of the aneurysm on $3 \mathrm{D}$ rotational angiography (width and height of the dome outside of additional blebs and daughter aneurysms). In general, on the basis of the rule of the manufacturer, the device was chosen adding $1 \mathrm{~mm}$ to the average width (to ensure good wall apposition) and subtracting $1 \mathrm{~mm}$ from the average height of the aneurysm (to adjust for the longitudinal increase caused by the horizontal compression). ${ }^{6}$ Starting in 2017, WEB devices were selected using a computer-based-simulation modeling tool (Sim\&Size software; Sim\&Cure; Grabels, France).

\section{Imaging Assessment}

Anatomic and angiographic results were independently evaluated by 2 interventional neuroradiologists not directly involved in patient treatment. A senior interventional neuroradiologist solved discrepancies.

Aneurysm occlusion and WEB shape modification were evaluated on the DSA performed at least at 12-month follow-up. The aneurysm occlusion rate was defined on the basis of the Raymond-Roy (RR) classification: complete occlusion (class 1), residual ostium (class 2 ), and residual aneurysm (class 3$)^{7}$

After WEB deployment, the size of the device was evaluated as "adequate" or "undersized." There were no cases of oversized devices resulting in WEB protrusion over the ostium. A detailed definition of undersized and adequately sized devices is reported in the On-line Appendix.

Aneurysm shape was dichotomized into regular (when the surface was smooth and regular in the $3 \mathrm{D}$ angiography) and irregular (in case of blebs or multilobular shape).

WEB shape change (also called "compaction") was defined as a decrease in the height of the device or a deepening of the proximal and distal concave recesses during follow-up: ${ }^{8}$ It was analyzed on nonsubtracted images. Methods to evaluate WEB changes are reported in the On-line Appendix.

\section{Statistical Analysis}

Categoric data were described by frequency, whereas quantitative data, by means and SDs. We assessed the following: 1) long-term angiographic aneurysm occlusion ("adequate occlusion" [RR 1/RR 2] versus "incomplete occlusion" [RR 3]); and 2) WEB shape modification (yes versus no) during midterm follow-up. A $\chi^{2}$ test was used to evaluate qualitative factors: vascular risk factors, location, ruptured status, bifurcation point versus sidewall, aneurysm shape, wide ostium ( $\geq 4$ versus $<4 \mathrm{~mm}$ ), vessel coming from the aneurysm, additional stent placement, WEB shape modification, immediate aneurysm occlusion, and correctly sized versus undersized devices. The $t$ test (2-tailed) was applied to assess quantitative factors (age, aneurysm dome size, dome/ostium ratio, aspect ratio). The independent variables significantly associated $(P \leq .1$ in the univariate analysis) with adequate occlusion or WEB shape modifications were analyzed together in a binary logistic regression to assess the independent contribution of each factor. The results of the regression model were calculated with the Wald test and expressed using a $P$ value and related odds ratio. All statistical analyses, descriptive and inferential, were performed with SPSS, Version 24 (IBM, Armonk, New York).

\section{RESULTS}

\section{Baseline Population and Aneurysm Characteristics}

In the 5-year period, 130 consecutive aneurysms/patients were treated with the WEB (detailed data in On-line Table 1). We extracted 86 patients (58 women, 28 men; mean age, 61 years; range, 35-76 years) available at midterm angiographic follow-up. Twenty-one patients (24\%) were treated in the setting of acute 

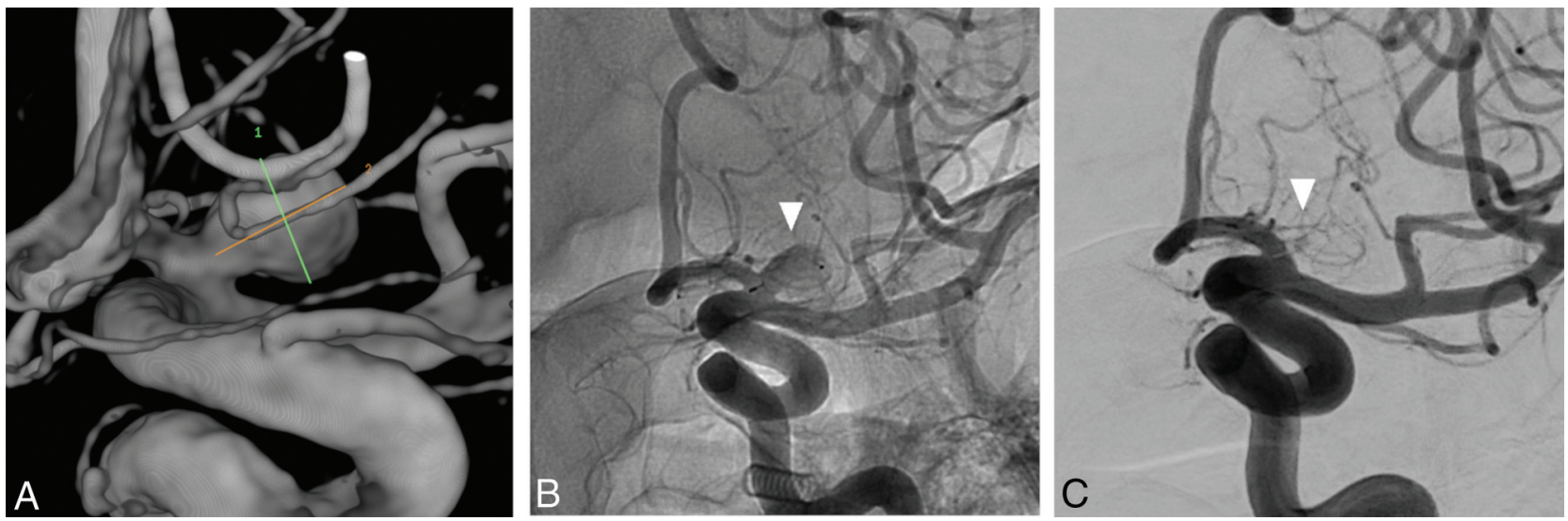

FIG 1. A, Left ICA angiography depicting an unruptured saccular aneurysm with regular morphology originating from the proximal $\mathrm{Al}$ segment. The aneurysm dome, height, and ostium diameters were 6,7 , and $3 \mathrm{~mm}$ (small ostium), respectively. Accordingly, this aneurysm had all the predicting factors of adequate occlusion. $B$, A WEB-SLS $(7 \mathrm{~mm}$ ) was opened inside the sac, with a good aneurysm wall apposition (white arrowhead). C, Twelve-month DSA follow-up shows complete occlusion of the aneurysm (white arrowhead).

SAH. The mean aneurysm dome size was $5.5 \pm 1.9 \mathrm{~mm}$; range, $3-11.5 \mathrm{~mm}$ ), and the most common location was the MCA bifurcation $(43 / 86=50 \%)$, followed by the basilar tip (BT) $(13 / 86=15 \%)$ and the anterior communicating artery $($ AcomA $)(12 / 86=13.9 \%)$.

\section{Treatment Characteristics and Technical Results}

The WEB-SL was used in all cases (On-line Table 2). The WEBSL and WEB-Single-Layer Sphere (SLS) were used in $90.6 \%$ $(78 / 86)$ and $8.4 \%(8 / 86)$ of patients, respectively. Additional stent placement was performed in 13 cases $(13 / 86=15 \%)$.

Successful WEB deployment was achieved in all cases. The mean intervention and fluoroscopy times were $66 \pm 17$ minutes (range, 50-103 minutes) and 30 12 minutes (range, 18-60 minutes), respectively.

\section{Angiographic Outcome of Aneurysms}

The mean angiographic follow-up was $17 \pm 11.5$ months; range, 12-32 months) (On-line Table 2). Immediately after treatment, RR 1/RR 2 occlusion was $48.8 \%$ (42/86). At 12-month follow-up, 49/86 (57\%) and 19/86 (22\%) aneurysms presented with complete occlusion and ostium remnants, respectively. The proportion of RR 1 and RR 2 occlusion was close to $49 \%$ and $33 \%$ at the 24-month follow-up (60 patients available), respectively. Overall, 51/86 (59\%) WEBs were defined as adequately sized, whereas 35/86 (41\%) were classified as undersized devices.

WEB shape change was detected among $22 \%$ of cases (19/ 86): $7 \%(6 / 86), 15 \%(13 / 86)$, and $0 \%(0 / 60)$ at 6-, 12-, and $24-$ month follow-up, respectively. Retreatment with additional stent and coiling was required in 11/86 recanalized aneurysms $(13 \%)$.

\section{Predictors of Adequate Occlusion and WEB Shape Change}

Significant predictors of occlusion and WEB shape change at univariate analysis were further analyzed in multivariable logistic regression (On-line Tables 3 and 4). Regular shape ( $O R=5.9$; 95\% CI , 1.4-24; $P=.01$ ) was an independent predictor of good occlusion, whereas a wide ostium $(\mathrm{OR}=0.2 ; 95 \% \mathrm{CI}, 0.01-1$; $P=.04)$ was an independent factor in incomplete occlusion.
Irregular shape $(\mathrm{OR}=5.4 ; 95 \% \mathrm{CI}, 1.4-19 ; P=.01)$ and a wide ostium $(\mathrm{OR}=9.8 ; 95 \% \mathrm{CI}, 1.6-60 ; P=.03)$ independently influenced the likelihood of WEB shape change at follow-up. Higher aneurysm dome size (continuous variable) showed a trend toward greater WEB shape modification $(\mathrm{OR}=1.39 ; 95 \%$ CI, 0.9-1.6; $P=.06)$.

\section{DISCUSSION}

\section{Predictors of Angiographic Occlusion}

Consistent with the literature of WEB devices reporting $80 \%$ adequate occlusion at 1 year, ${ }^{3,9}$ in our series, RR 1/RR 2 occlusion was $79 \%$ and $81 \%$ after 12 - and 24-month DSA follow-up. Kabbasch et al, ${ }^{10}$ evaluating a series of 98 aneurysms treated with the WEB, reported dome size, intra-aneurysmal thrombosis, and additional coiling as factors associated with lower occlusion at 6-month follow-up. Another recent series of 24 aneurysms reported that ostium size and dome-to-ostium ratio were significantly related to the angiographic results after WEB treatment. ${ }^{2}$ To our knowledge, there are no studies investigating independent predictors of occlusion after treatment with WEB devices. In our series of 86 patients, eliminating the influence of potential confounders using the regression model showed that ostium diameter and aneurysm shape were the only independent predictors of occlusion. Among the subgroup of adequately occluded aneurysms, $60 \%$ had a diameter of the ostium $<4 \mathrm{~mm}$ (Fig 1), whereas $40 \%$ had a diameter of the ostium $\geq 4 \mathrm{~mm}$. In addition, wide-ostium lesions represented $91 \%$ of the incompletely occluded aneurysms (Fig 2). Therefore, the binary logistic regression revealed that a wide ostium indicated $80 \%$ reduction in the probability of achieving long-term good occlusion after WEB treatment $(\mathrm{OR}=0.2$; 95\% CI , 0.01-1; $P=.04)$.

Lower occlusion rates among lesions with a wide ostium are likely related to the following reasons: 1) Ostium coverage might be more difficult among large-ostium lesions; and 2) the higher hemodynamic impact of blood flow may decrease the intrasaccular thrombosis or may promote WEB displacement toward the aneurysm fundus. Hemodynamic studies showed that intra- 

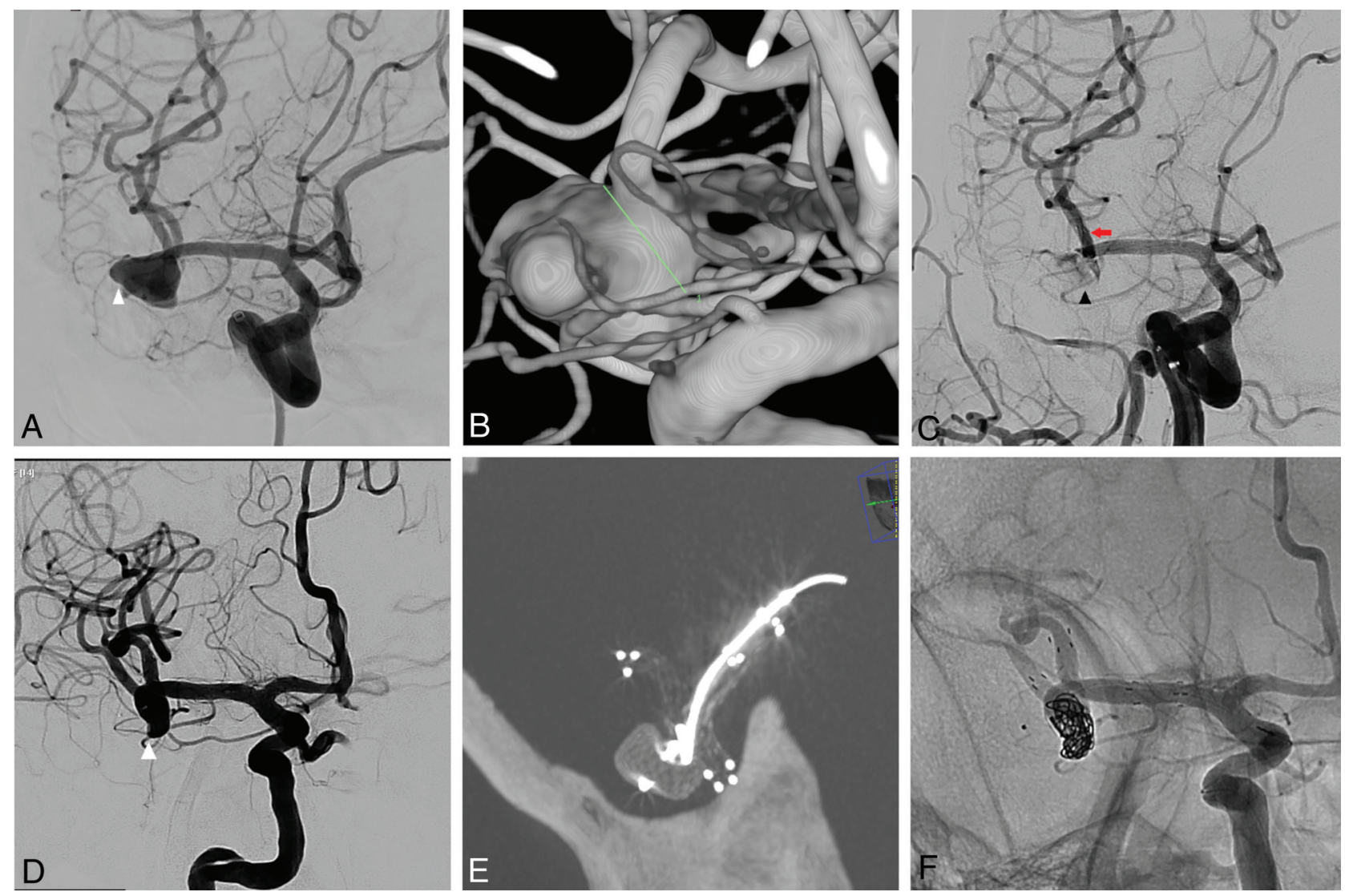

FIG 2. A, Right ICA angiography showing an irregular 9 (dome) $\times 5 \mathrm{~mm}$ (height) MCA unruptured aneurysm (white arrowhead) with a wide ostium $(6 \mathrm{~mm})(B)$. C, A WEB-SL $(10 \times 5)$ was delivered inside the sac (black arrowhead). Subsequently, in the same treatment session, a Neuroform Atlas (Stryker Neurovascular, Kalamazoo, Michigan) $3 \times 20$ (red arrow) was implanted from the M1 to the M2 (superior division branch) due to a small WEB protrusion into the vessel. D. Twelve-month DSA follow-up shows aneurysm recanalization (white arrowhead). This aneurysm presented with all the risk factors (wide ostium and irregular shape) for incomplete occlusion. E, Flat panel CT reconstruction depicts WEB shape modification (compaction) and the deployment of a second Neuroform Atlas $3 \times 20$ with a jailed microcatheter inside the sac. $F$, The residual aneurysm was completely occluded after $Y$-stent placement assisted coiling.

aneurysmal blood flow was faster when the aneurysm neck was wider. $^{11}$ The presence of a wide neck has been previously reported as a factor associated with lower occlusion rates after coiling. ${ }^{12}$ Accordingly, higher intra-aneurysmal blood flow among lesions with a wide ostium may contribute, at least in theory, to the lower occlusion rate after WEB treatment. When one investigates the literature about other treatment options, a wide ostium appears significantly associated with aneurysm recanalization after coiling, ${ }^{13}$ whereas aneurysm size and incorporation of a branch vessel predicted persistence after flow diversion. ${ }^{14}$ Most interesting, in our analysis, aneurysm size and vessels coming from the aneurysm were not predictors of recanalization after WEB treatment. It is likely that size did not influence the angiographic occlusion due to the relatively small range of aneurysm dome size in our study (from 3 to $11 \mathrm{~mm}$ ). Conversely, Bender et $\mathrm{al}^{14}$ reported a larger size as a predictor of lower occlusion after flow diversion, including a larger range of aneurysm diameters (from 1 to $31 \mathrm{~mm}$ ). Finally, the distal hemodynamic demand through the branching vessel will likely maintain the flow across the ostium, lowering the occlusion in case of flow-diverter devices, but not impacting intrasaccular thrombosis after WEB implantation.
The second independent predictor of occlusion was aneurysm shape: Aneurysms with regular morphology were 6 times more likely to be occluded at 12-month follow-up (Fig 1), compared with lesions presenting with an irregular sac $(\mathrm{OR}=5.9$; 95\% CI, 1.4\%-24\%; $P=.01$ ) (Fig 2). Our study is the first highlighting shape as prognosticator of occlusion after the WEB treatment. In our experience, among irregular lesions, the more difficult selection of the appropriate WEB dimension, together with the incomplete apposition of the device on the aneurysm wall and ostium, may decrease the likelihood of good occlusion.

Although undersized WEBs were more represented among the group of incompletely occluded aneurysms (58\% versus $34 \%$ ), adequate sizing and undersizing were not significant predictors of the angiographic outcome. However, most of the WEBs were just slightly undersized, often in case of branching vessels coming from the aneurysm. Accordingly, the impact of this factor can be underestimated in our series. However, other authors also found similar results: Herbreteau et $\mathrm{al}^{15}$ in a prospective series of 39 aneurysms treated with WEB, reported statistically comparable rates of complete occlusion among undersized and appropriately sized WEB devices. 

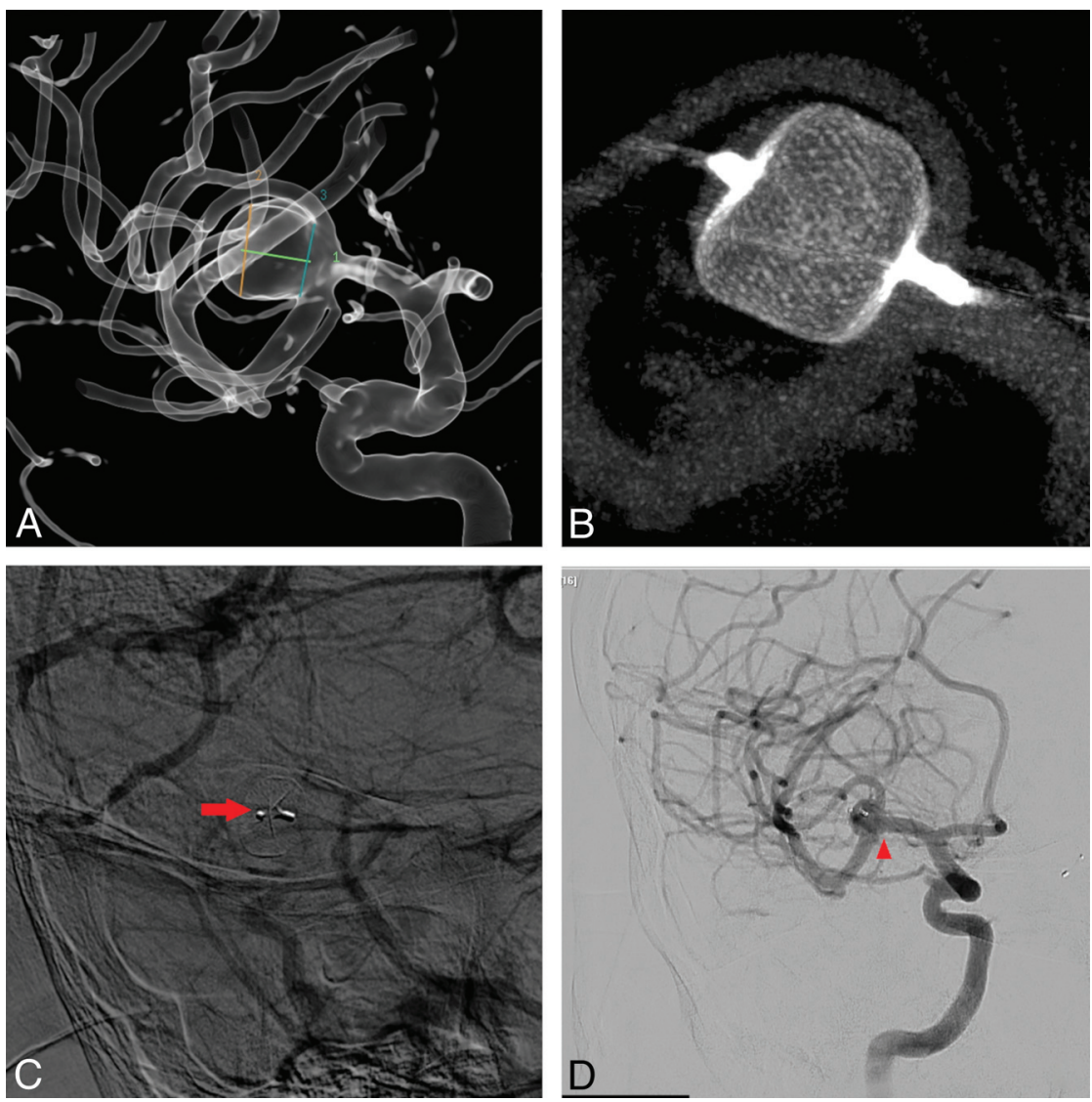

FIG 3. A, Right MCA saccular and unruptured aneurysm (dome size, $8 \mathrm{~mm}$; ostium size, $5.5 \mathrm{~mm}$; height, $7 \mathrm{~mm}$ ). $B$, The aneurysm was treated with a WEB-SL $9 \times 5$, and the flat panel CT reconstruction showed the correct WEB deployment inside the sac. $C$, The WEB presented with an evident reduction of the height at the 14-month DSA angiography follow-up (red arrow). D, However, despite the evident WEB shape modification, the aneurysm was still adequately occluded, having a residual ostium (red arrowhead).

Another important point was that WEB shape change, though higher among the incompletely occluded aneurysms ( $50 \%$ versus $17.5 \%)$, was not an independent prognosticator of occlusion in the multivariate model (Fig 3). As discussed in the following paragraph, these data corroborate the theory that WEB shape modifications (sometimes called "WEB compaction") are more of an epiphenomenon of the aneurysm thrombosis process rather than a reflection of the treatment failure.

Finally, consistent with recent publications, ${ }^{16}$ the use of the WEB with stent placement was feasible and effective, but additional devices did not significantly influence the final occlusion rate: Ruptured and unruptured lesions presented comparable rates of good angiographic results.

\section{Predictors of WEB Shape Modification}

WEB shape modification has been called, in some reports, "compression" or "compaction," and it is relative to the reduction of the height of the WEB on the angiographic follow-up. ${ }^{4}$ This feature has been reported in up to $65 \%$ of patients, and whether WEB compaction is directly associated with aneurysm recanalization is still debated. ${ }^{1,2}$ Recent series reported similar rates of adequate occlusion, among the group of lesions both with and without WEB modifications. ${ }^{2,4}$ Herbreteau et $\mathrm{al}^{15}$ reported WEB shape modification among $31 \%$ of aneurysms, stressing the absence of a relationship between the sizing of the device and its height reduction. The WEB shape change was associated with an increase of the ostium remnants, without impacting the overall rate of adequate occlusion. Our results were in accordance with this study, showing no statistically significant correlation between sizing and the risk of WEB shape modification at follow-up. Most interesting, aneurysm shape and ostium dimensions were significant predictors of WEB compaction. Irregular aneurysms were $63 \%$ and $28 \%$ among the group of lesions with and without WEB shape modification, respectively, increasing the probability of this phenomenon at follow-up $(\mathrm{OR}=5.4$; 95\% CI, 1.4-19; $P=.01$ ) (On-line Table 4) $>5$ times, while the presence of a wide ostium increased the likelihood of compaction $(\mathrm{OR}=$ 8; $95 \%$ CI, $1.6-60 ; P=.03$ ) by 10 times.

However, this phenomenon is still a matter of debate. First, as reported in the article of Cognard and Januel, ${ }^{17}$ and in the response to this article by Pierot, ${ }^{8}$ the term "compression" is probably not appropriate because it indicates only a "waterhammer" mechanism that is not yet demonstrated. Pierot et al ${ }^{3}$ suggested that clot organization and retraction may contribute to both the intrasaccular occlusion and the shrinkage of the WEB. This effect has also been described in experimental models of coiled aneurysms, which observed that fibrosis increased coil retraction and compaction. ${ }^{18}$ However, we still do not have a full explanation for this event, and mechanisms underlying WEB compaction are probably multifactorial. In our series, we demonstrated that WEB shape modifications were not associated with lower aneurysm occlusion, supporting the theory of a retraction of the marker recesses related to thrombus evolution/ fibrosis.

Reasons behind the association between irregular shape and WEB shape change are difficult to explain, and it has never been investigated before. Cognard and Januel, ${ }^{17}$ having reported this phenomenon for the first time, stressed that a large ostium and irregular shape were likely the main reasons for aneurysm remnants and cage compaction in their series. The authors questioned whether the cage must occupy the entire volume to obtain complete and stable occlusion, minimizing the risk of cage modifications by clot formation or cage displacement. ${ }^{19}$

Finally, as we previously reported, WEB shape modification was unlikely to be observed among small-ostium lesions $(<4$ 
$\mathrm{mm}$ ), while a wide ostium was independently associated with this phenomenon. Accordingly, hemodynamic factors related to a larger diameter of the ostium may at least participate in the complex mechanism leading the decrease of the height of the device. $^{20}$

\section{Limitations of the Study}

Our study has limitations intrinsic to single-center series. The data were analyzed retrospectively, and the number of patients was relatively small. The WEB shape modification was not quantitatively evaluated. Finally, due to the relatively small number of patients, some variables may not reach statistical significance in the multivariate analysis.

\section{CONCLUSIONS}

In our study, approximately $80 \%$ of aneurysms were adequately occluded after WEB implantation, whereas nearly $20 \%$ of the devices showed shape modification at follow-up. The likelihood of good occlusion was 5 times less in the presence of a wide ostium, whereas aneurysms with regular morphology were about 6 times more likely to be occluded. WEB shape change, though more prevalent among incompletely occluded lesions, was not independently associated with angiographic occlusion, and it was strongly dependent on aneurysm shape and ostium size.

Disclosures: Pierre-Henri Lefevre-UNRELATED: Payment for Development of Educational Presentations: Medtronic. Alain Bonafe-UNRELATED: Consultancy: Medtronic, Stryker, MicroVention.* Vincent Costalat-UNRELATED: Consultancy: Balt, Medtronic, MicroVention, Balt Stryker, Cerenovus; Grants/Grants Pending: Stryker, Medtronic; Payment for Development of Educational Presentations: Stryker, Medtronic. *Money paid to institution.

\section{REFERENCES}

1. Pierot L, Moret J, Barreau X, et al. Safety and efficacy of aneurysm treatment with WEB in the cumulative population of three prospective, multicenter series. J Neurointerv Surg 2018;10:553-59 CrossRef Medline

2. Limbucci N, Leone G, Rosi A, et al. Endovascular treatment of unruptured intracranial aneurysms by the Woven EndoBridge device (WEB): are there any aspects influencing aneurysm occlusion? World Neurosurg 2018;109:e183-93 CrossRef Medline

3. Pierot L, Gubucz I, Buhk JH, et al. Safety and efficacy of aneurysm treatment with the WEB: results of the WEBCAST 2 study. AJNR Am J Neuroradiol 2017;38:1151-55 CrossRef Medline

4. Janot K, Herbreteau D, Amelot A, et al. Quantitative evaluation of WEB shape modification: a five-year follow-up study. $J$ Neuroradiol 2019 Mar 7. [Epub ahead of print] CrossRef Medline

5. Pierot L, Biondi A, Narata AP, et al. Should indications for WEB aneurysm treatment be enlarged? Report of a series of 20 patients with aneurysms in "atypical" locations for WEB treatment. $J$ Neuroradiol 2017;44:203-09 CrossRef Medline

6. Lescher S, Du Mesnil de Rochemont R, Berkefeld J. Woven Endobridge (WEB) device for endovascular treatment of complex unruptured aneurysms: a single center experience. Neuroradiology 2016;58:383-90 CrossRef Medline

7. Roy D, Milot G, Raymond J. Endovascular treatment of unruptured aneurysms. Stroke 2001;32:1998-2004 CrossRef Medline

8. Pierot L. Letter: WEB aneurysm treatment: occlusion stability and “compression." Neurosurgery 2015;77:E666-67 CrossRef Medline

9. Asnafi S, Rouchaud A, Pierot L, et al. Efficacy and safety of the Woven EndoBridge (WEB) device for the treatment of intracranial aneurysms: a systematic review and meta-analysis. AJNR Am J Neuroradiol 2016;37:2287-92 CrossRef Medline

10. Kabbasch C, Goertz L, Siebert E, et al. Factors that determine aneurysm occlusion after embolization with the Woven EndoBridge (WEB). J Neurointerv Surg 2019;11:503-10 CrossRef Medline

11. Tateshima S, Chien A, Sayre J, et al. The effect of aneurysm geometry on the intra-aneurysmal flow condition. Neuroradiology 2010; 52:1135-41 CrossRef Medline

12. Hope JK, Byrne JV, Molyneux AJ. Factors influencing successful angiographic occlusion of aneurysms treated by coil embolization. AJNR Am J Neuroradiol 1999;20:391-99 Medline

13. Tan IY, Agid RF, Willinsky RA. Recanalization rates after endovascular coil embolization in a cohort of matched ruptured and unruptured cerebral aneurysms. Interv Neuroradiol 2011;17:27-35 CrossRef Medline

14. Bender MT, Colby GP, Lin LM, et al. Predictors of cerebral aneurysm persistence and occlusion after flow diversion: a single-institution series of $\mathbf{4 4 5}$ cases with angiographic follow-up. J Neurosurg 2018;130:259-67 CrossRef Medline

15. Herbreteau D, Bibi R, Narata AP, et al. Are anatomic results influenced by WEB shape analysis in a prospective, single-center series of 39 patients with aneurysms treated with the WEB. AJNR Am J Neuroradiol 2016;37:2280-86 CrossRef Medline

16. Cagnazzo F, Ahmed R, Dargazanli C, et al. Treatment of wideneck intracranial aneurysms with the Woven EndoBridge device associated with stenting: a single-center experience. AJNR Am J Neuroradiol 2019;40:820-26 CrossRef Medline

17. Cognard C, Januel AC. Remnants and recurrences after the use of the WEB intrasaccular device in large-neck bifurcation aneurysms. Neurosurgery 2015;76:522-30; discussion 530 CrossRef Medline

18. Rouchaud A, Brinjikji W, Dai D, et al. Autologous adipose-derived mesenchymal stem cells improve healing of coiled experimental saccular aneurysms: an angiographic and histopathological study. J Neurointerv Surg 2018;10:60-65 CrossRef Medline

19. Cognard C, Januel AC. In reply: WEB aneurysm treatment: occlusion stability and “compression.” Neurosurgery 2015;77:E667-69 CrossRef Medline

20. Caroff J, Mihalea C, Da Ros V, et al. A computational fluid dynamics (CFD) study of WEB-treated aneurysms: can CFD predict WEB “compression” during follow-up? J Neuroradiol 2017;44:262-68 CrossRef Medline 